\title{
Supporting Knowledge Transfer for Low Vision Interventions Through an Online Directory of Validated Assessment Tools
}

\author{
Walter Wittich, \\ PhD FAAO CLVT \\ Associate Professor, \\ School of Optometry \\ Université de Montréal
}

Catherine Houtekier, M.L.I.S. Planning, Programming and Research Officer CRIR/Nazareth and LouisBraille Institute of the CISSS of Montérégie-Centre

Sylvie Cantin, M.A

Planning, Programming and Research Officer CRIR/Nazareth and LouisBraille Institute of the CISSS of Montérégie-Centre

\begin{abstract}
Research Letter
The choice of outcome measures for large-scale population-based or clinical studies in Canada that contain objective and subjective assessments of visual function, such as the Canadian Longitudinal Study on Aging (CLSA) ${ }^{1}$ or the Comprehensive Assessment of Neurodegeneration and Dementia (COMPASS-ND) Study ${ }^{2}$, is limited by the availability of assessment tools that have been validated in both official languages. Similarly, vision healthcare professionals lack the time to search the research literature for validated outcome measures they can use with both anglophone and francophone patients, which makes meaningful comparisons difficult. This challenge is even more pronounced in clinical sub-specialities such as low vision. Meanwhile, researchers direct their publications mainly to an academic audience, and rarely present recent discoveries in a format ready for uptake in the clinical environment ${ }^{3}$.
\end{abstract}

In an effort to further bridge this gap, we present an online directory promoting the adoption of validated research tools in clinical practice that are suitable for persons presenting with a visual impairment, and that are available in French, or in French and English. ORVIS (Outils pour la Réadaptation de la VISion / DirectORy of Tools for VISion Rehabilitation, www.orvis.vision) is a database of measurement tools for which the validity and fidelity have been or are in the process of being evaluated ${ }^{4}$. Its purpose is to facilitate knowledge transfer by integrating research tools into clinical practice, and to address a clinical need. These tools promote the rigorous and reliable appraisal of characteristics relevant to the assessment, treatment and rehabilitation of visual impairment, and include functional, perceptual, cognitive and psychological evaluation instruments. Each tool is presented with a descriptive sheet that identifies the tool and its metrological characteristics, and lists bibliographical references of the scientific studies consulted. The project management team includes a researcher, who is responsible for the infrastructure (funded in part by the FRQ-S Vision Health Research Network: http://visionnetwork.ca/), two research professionals, an information professional and a documentation technician from the Institut Nazareth et Louis-Braille du CISSS de la Montérégie-Centre. Two clinicians from the Institut are added to the project team and form an advisory committee to ensure ongoing research-clinic linkage and relevance.

As of December 2019, 29 tool summaries were available free of charge. The consultation statistics reflect the growing interest generated by the directory (see Figure 1). Since it went online in November 2015, www.orvis.vision has been visited 16,867 times by 10,319 unique visitors from more than 15 countries, most of whom are part of the Francophonie (see Figure 2). A dedicated e-mail address allows Internet users to send requests for information directly to the project team. In light of these results, www.orvis.vision intends to pur- 
sue its work by focusing on three objectives: 1) to continue choosing tools to be documented for knowledge transfer into clinical low vision rehabilitation research; 2) to produce validated French translations of certain relevant tests or measurement scales that are currently available only in English; and 3) to promote the directory more widely. Ultimately, the ORVIS team aims to strengthen evidence-based vision rehabilitation practice while promoting integrated research that includes participants and clients, inclusively spanning both official languages.

Figure 1

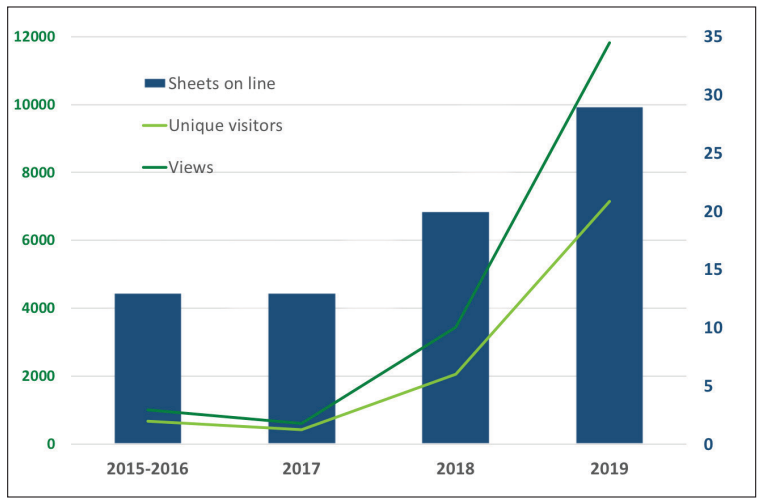

Figure 2

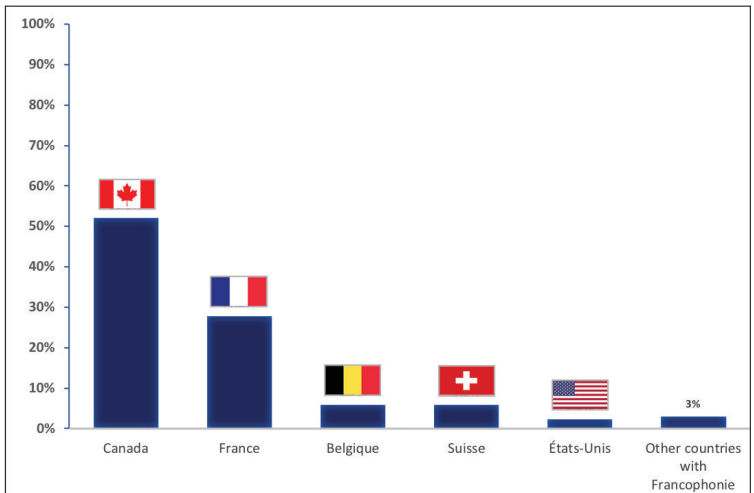

AUTHOR CREDIT STATEMENT:

Walter Wittich: Conceptualization, Methodology, Investigation, Writing - original draft, Supervision, Funding acquisition; Sylvie Cantin: Conceptualization, Methodology, Validation, Investigation, Formal analysis, Resources, Data retention, Writing - revision and editing, Project administration; Catherine Houtekier: Conceptualization, Methodology, Validation, Formal analysis, Investigation, Resources, Data retention, Writing - revision and editing, Visualization, Project Administration.

KEY WORDS:

low vision, rehabilitation, assessment

CORRESPONDING AUTHOR:

Walter Wittich, walter.wittich@umontreal.ca

ACKNOWLEDGEMENTS:

This work is funded by an FRQ-S Vision Health Research Network infrastructure grant, and supported by the Fondation EnVue of the Institut Nazareth et Louis-Braille du CISSS de la Montérégie-Centre

DECLARATIONS OF INTEREST:

none

\section{REFERENCES}

1. Raina P, Wolfson C, Kirkland SA, et al. The Canadian Longitudinal Study on Aging (CLSA). Can J Aging 2009;28:221-9. https://doi. org/10.1017/S0714980809990055.

2. Chertkow H, Borrie M, Whitehead V, et al. The comprehensive assessment of neurodegeneration and dementia: Canadian cohort study. Can J Neurol Sci 2019;46:499-511. https://doi.org/10.1017/ cjn.2019.27.
3. Markowitz SN. State-of-the-art: low vision rehabilitation. Can J Ophthalmol / J Can d'Ophtalmologie 2016;51:59-66. https://doi. org/10.1016/j.jcjo.2015.11.002.

4. Wittich W. ORVIS: a directory of tools for vision rehabilitation. Ann Eye Sci 2018;3:AB106 